\title{
Diagnosis-specific effect of familial loading on verbal working memory in schizophrenia
}

\author{
David Zilles · Sarah Burke - Thomas Schneider-Axmann • \\ Peter Falkai $\cdot$ Oliver Gruber
}

Received: 22 August 2008/ Accepted: 29 January 2009/Published online: 10 March 2009

(C) The Author(s) 2009. This article is published with open access at Springerlink.com

\begin{abstract}
Background Working memory disturbances are a frequently replicated finding in schizophrenia and less consistent also in schizoaffective disorder. Working memory dysfunctions have been shown to be heritable and have been proposed to represent a promising endophenotype of schizophrenic psychoses.

Methods In the present study, we investigated the effects of familial loading on performance rates in circuit-specific verbal and visuospatial working memory tasks in matched samples of schizophrenic patients (from multiply affected or uniaffected families), schizoaffective patients (from multiply affected or uniaffected families), and healthy subjects.

Results We found a significant interaction effect between familial loading and diagnosis in terms of a diagnosisspecific detrimental effect of familial loading on the performance of schizophrenic (but not schizoaffective) patients in the articulatory rehearsal task.

Conclusion This finding of a circuit-specific verbal working memory deficit in schizophrenic patients with additional familial loading is consistent with prior studies, which provided evidence for the existence of specific subgroups of schizophrenic patients with selective working memory impairments and for diagnosis-specific dysfunctions of the articulatory rehearsal mechanism in schizophrenic, but not in schizoaffective patients. Together, these findings suggest that the genetic risk for (a subtype of) schizophrenia may be associated with dysfunctions of the
\end{abstract}

D. Zilles · S. Burke $\cdot$ T. Schneider-Axmann · P. Falkai ·

O. Gruber $(\square)$

Department of Psychiatry and Psychotherapy,

Georg August University, Von-Siebold-Str. 5,

37075 Göttingen, Germany

e-mail: ogruber@gwdg.de brain system, which underlies the articulatory rehearsal mechanism, the probably phylogenetically youngest part of human working memory.

Keywords Intermediate phenotype - Biological marker . Genetic loading · Genotype · Neuroimaging

\section{Introduction}

Cognitive dysfunction is considered as a core deficit of schizophrenia. Neuropsychological impairments are found in a multitude of different cognitive domains [11]. In particular, impaired functioning of verbal as well as visuospatial working memory processes is a frequently replicated finding in schizophrenic patients [4, 8], and is already present in first episode patients [1]. Yet, a problem in the investigation of working memory processes is the multitude of different tests all expected to examine working memory functioning which leads to a lack of specificity and comparability. Recent functional neuroimaging studies in healthy subjects using more refined paradigms from experimental psychology have identified different neuronal networks, which underlie specific verbal and visuospatial working memory functions in humans [14, 15]. According to an evolutionary-based model [16], human working memory is suggested to consist of two different brain systems with different evolutionary origin: a presumably phylogenetically older, multimodal system which is also present in non-human primates, and a second, humanspecific speech-based system (the verbal rehearsal mechanism), which may have emerged later in the course of human speech development. Subsequent clinical studies using the same paradigms as in the previous fMRI studies revealed impaired working memory performance of 
schizophrenic patients in comparison to a matched group of healthy controls [17]. On the other side, a high interindividual variability was observed within the patient group with, in part, isolated deficits in single tasks, e.g., in a verbal working memory task which tapped on the articulatory rehearsal mechanism or in two visuospatial tasks, respectively. Based on the prior fMRI investigations and on lesion studies, which provided additional confirmatory evidence for the association of brain structures activated during fMRI with distinct sub-functions of working memory, these task-specific deficits can be interpreted to represent specific disturbances of the identified neuronal networks in schizophrenic patients [17].

There is also evidence from a recent meta-analysis suggesting that working memory impairments represent specific deficits that are independent of measures of general cognitive ability such as IQ [9]. Furthermore, working memory deficits have been proposed as a potential endophenotype of schizophrenic psychoses [13]. Endophenotypes are thought to be under a strong and more direct genetic influence than the complex and statedependent symptoms of the disease and there is indeed a growing number of studies reporting associations between genetic polymorphisms and cognitive functioning. For instance, Spellmann et al. [22] recently reported associations between specific polymorphisms of the SNAP-25 gene and performance in several neuropsychological tests of verbal memory and executive functions in a sample of schizophrenic patients.

Because of the assumed genetic influences, endophenotypic candidate markers must show heritability, which means that they should be observable (at least to some extent) even in unaffected relatives of schizophrenic patients. In fact, recent studies have revealed a significantly reduced performance of healthy relatives of schizophrenic patients in several working memory tasks $[2,5]$. In the same context, studies investigating working memory performance in dependence of the grade of familial or genetic loading are also of great interest. Cannon [3] found that spatial working memory functioning discriminates between healthy monozygotic twins of schizophrenic patients, healthy dizygotic twins of schizophrenic patients and healthy control twins. There was a linear decrease in performance with increasing genetic risk for schizophrenia. Glahn et al. [10] investigated spatial working memory functioning in healthy co-twins of schizophrenic patients. Monozygotic and dizygotic co-twins as well as control twins without familial loading were tested. The authors reported that the performance in a spatial delayed-response task decreased with increasing liability for schizophrenia. Monozygotic co-twins of affected patients performed worse than dizygotic co-twins, who performed worse than control twins without familial loading [10]. Tuulio-Henriksson [24] assessed the performance of healthy siblings from families with one schizophrenia patient versus multiply affected families and found an association between the number of affected relatives and increasing deficits in a backward visual span task.

The literature with respect to working memory deficits in schizoaffective disorder is less extensive. However, impaired spatial working memory has also been reported in schizoaffective patients [12]. There is a recent and continuing debate whether the diagnosis schizoaffective disorder constitutes an independent disease entity [19] and if the underlying etiology, pathogenesis and neurobiology is different from schizophrenia on the one hand and affective disorders on the other hand. In a recent study, which directly compared working memory performance in matched groups of schizophrenic and schizoaffective patients, it could be demonstrated that the performance in a verbal working memory task imposing demands on the articulatory rehearsal mechanism differed between the patient groups with schizophrenic patients showing significantly worse performance [18]. This finding suggests that the neurocognitive and/or neurobiological basis of schizophrenia and schizoaffective disorder may, at least in part, be different.

In the present study, we sought to investigate the influence of familial (genetic) loading on performance in these specific working memory tasks, i.e., the functioning of the underlying neural networks, in samples of subjects with schizophrenia and schizoaffective disorder from multiply affected and uniaffected families, respectively. According to the endophenotype criteria, which include heritability and co-segregation of endophenotype and illness, we hypothesized that patients from multiply affected families would show worse performance in verbal and visuospatial working memory tasks as compared to patients from uniaffected families due to the higher degree of genetic loading. Moreover, in the light of the prior finding of significant group differences in verbal working memory functioning between schizophrenic and schizoaffective patients [18], which may possibly indicate a different genetic basis for these two diagnostic categories, we sought to further clarify whether the hypothesized familial loading effect on the verbal component of working memory would also show diagnostic specificity for schizophrenia (vs. schizoaffective disorder).

\section{Methods}

Subjects

Inclusion criteria for patients were diagnosis of schizophrenia or schizoaffective disorder according to ICD-10 and DSM-IV criteria and age range from 18 to 65 years. 
Exclusion criteria were acute suicidality, involuntary treatment, current substance abuse, history of brain trauma, diseases with alterations of cerebral metabolism, uncorrected visual or auditory disability and mental retardation. Criteria for the recruitment of the healthy comparison subjects were the same as for patients plus the absence of any past or present psychiatric disorder.

In total, 16 schizoaffective patients, 68 schizophrenic patients and 59 healthy control subjects were tested with the circuit-specific working memory tasks described below. Instead of using multivariate regression analyses in the total sample in a 'post hoc' attempt to statistically dissect the (possibly interacting) effects of different intervening factors, we preferred a more straightforward approach to use only small, but carefully matched samples in the statistics. As familial loading was found to be present in only five schizoaffective and eight schizophrenic patients, this determined and restricted the sample size in each subgroup. All patients were inpatients and were recruited at the Department of Psychiatry and Psychotherapy, Saarland University Hospital. Healthy controls were recruited among friends and family members of our laboratory staff, so we were able to rule out relevant psychiatric disorders without an extensive screening procedure. Approval by a local ethics committee was obtained, and all subjects gave written informed consent.

After a careful matching procedure, 10 schizoaffective patients (5 with vs. 5 without familial loading), 16 schizophrenic patients ( 8 with vs. 8 without familial loading), and 13 control subjects (5 matched to the schizoaffective subgroups and 8 matched to the schizophrenic subgroup) remained in the sample for statistical analyses. At the time of the experimental neuropsychological testing, all of these 26 patients were treated with antipsychotic medication, mostly second generation antipsychotics. Some of the schizoaffective patients were additionally treated with mood stabilizers or antidepressants. The clinical state of the patients was assessed at the day of the experiment using clinical global impression scale (CGI), Montgomery Asberg depression scale (MADRS), positive and negative syndrome scale (PANSS).

\section{Experimental tasks}

Experimental neuropsychological testing was performed under standardized laboratory conditions. The behavioral experiment consisted of four tasks, testing for different types of working memory functions, i.e., articulatory rehearsal, non-articulatory maintenance of phonological information (under articulatory suppression), visuospatial rehearsal and the maintenance of visuospatial patterns (under visuospatial suppression). The order of the tasks was counterbalanced across subjects.
At the beginning of each task, a $5 \times 5$ matrix appeared on the screen for $2 \mathrm{~s}$ with four squares of the matrix filled with phonologically similar letters. Dependent on the respective task, subjects had to remember either the phonological identity ("sound") of the depicted letters, or the positions of the letters within the matrix. During a 4-s delay interval, subjects had to perform the respective memory strategy that had been practiced prior to the task. In the response phase, a single letter was depicted in one of the squares of the matrix for $1 \mathrm{~s}$. In the verbal working memory trials, the subjects had to decide whether this probe matched one of the target letters. In the visuospatial working memory conditions, the subjects had to judge whether the same square had been filled during target presentation. For a more detailed description of the experimental paradigms and the task instructions see $[15,18]$.

Statistical analyses of the performance rates in these working memory tasks were carried out using SPSS for Windows (version 16.0). The respective statistical tests are described in more detail in the following sections.

\section{Results}

Demographic variables

Working memory performance rates of 39 subjects (schizophrenia $n=16$, schizoaffective disorder $n=10$, healthy controls $n=13$ ) were included in the statistical analyses of familial loading effects (see Sect. "Methods"). Both patient groups were subdivided into groups with and without familial loading, respectively. The demographic variables are displayed in Table 1 , and working memory task performance rates of each group in Table 2 .

\section{Analyses of possible intervening variables}

To detect possible confounding variables, we analyzed the larger sample of healthy controls $(n=59)$. Pearson's product moment correlations were computed between working memory performance rates, on one side, and age and years of education, on the other. One-way analysis of variance was conducted to analyze if there were gender effects on working memory performance rates. In both the verbal and visuospatial rehearsal tasks, we observed a significant positive correlation between performance and years of education (articulatory rehearsal: $r=0.37$, $P=0.004$; visuospatial rehearsal: $r=0.42, P=0.001$ ) and a significant negative correlation between performance and age (articulatory rehearsal: $r=-0.28, P=0.033$; visuospatial rehearsal: $r=-0.36, P=0.005)$. There were 
Table 1 Demographic variables [mean (standard deviation)]

\begin{tabular}{|c|c|c|c|c|c|}
\hline & $\begin{array}{l}\text { Schizophrenia with } \\
\text { genetic loading }\end{array}$ & $\begin{array}{l}\text { Schizophrenia without } \\
\text { genetic loading }\end{array}$ & $\begin{array}{l}\text { Schizoaffective with } \\
\text { genetic loading }\end{array}$ & $\begin{array}{l}\text { Schizoaffective without } \\
\text { genetic loading }\end{array}$ & Controls \\
\hline$n$ & 8 & 8 & 5 & 5 & 13 \\
\hline Age (years) & $41.9(9.4)$ & $37.1(7.4)$ & $35.8(5.3)$ & $42.6(10.1)$ & $38(9.6)$ \\
\hline Education (years) & $16.2(4.2)$ & $14.7(3.5)$ & $13.2(2.8)$ & $13.6(3.1)$ & $14.3(2.3)$ \\
\hline Disease duration (years) & $13.4(9.9)$ & $7.0(3.5)$ & $8.0(4.0)$ & $14.0(7.1)$ & \\
\hline Gender (m/f) & $7 / 1$ & $6 / 2$ & $2 / 3$ & $2 / 3$ & $9 / 4$ \\
\hline
\end{tabular}

Table 2 Accuracy in the four working memory tasks

\begin{tabular}{|c|c|c|c|c|c|c|c|c|c|c|c|c|c|c|c|}
\hline & \multicolumn{3}{|c|}{ Controls } & \multicolumn{3}{|c|}{$\begin{array}{l}\text { Schizophrenia } \\
\text { with genetic } \\
\text { loading }\end{array}$} & \multicolumn{3}{|c|}{$\begin{array}{l}\text { Schizophrenia } \\
\text { without } \\
\text { genetic } \\
\text { loading }\end{array}$} & \multicolumn{3}{|c|}{$\begin{array}{l}\text { Schizoaffective } \\
\text { with genetic } \\
\text { loading }\end{array}$} & \multicolumn{3}{|c|}{$\begin{array}{l}\text { Schizoaffective } \\
\text { without genetic } \\
\text { loading }\end{array}$} \\
\hline & $n$ & $m$ & SD & $n$ & $m$ & SD & $n$ & $m$ & SD & $n$ & $m$ & SD & $n$ & $m$ & SD \\
\hline Articulatory rehearsal & 13 & 93.7 & 4.96 & 8 & 85.4 & 6.54 & 8 & 94.0 & 5.68 & 5 & 93.2 & 3.02 & 5 & 90.2 & 5.87 \\
\hline Non-articulatory maintenance of phonological information & 13 & 87.5 & 6.09 & 8 & 79.6 & 9.42 & 8 & 89.0 & 6.38 & 5 & 84.6 & 4.85 & 5 & 85.9 & 8.05 \\
\hline Visuospatial rehearsal & 13 & 94.3 & 4.53 & 8 & 79.6 & 13.3 & 8 & 90.3 & 5.67 & 5 & 85.9 & 8.53 & 5 & 89.4 & 6.31 \\
\hline Visuospatial pattern maintenance & 13 & 90.7 & 5.61 & 8 & 75.2 & 10.6 & 8 & 86.3 & 6.36 & 5 & 81.1 & 11.8 & 5 & 74.9 & 14.0 \\
\hline
\end{tabular}

$n$ sample size, $m$ mean percentage of correct answers, $S D$ standard deviation

no significant influences of gender on working memory performance rates.

\section{Analyses of the effects of familial loading}

Subsequently, we performed group comparisons as a 5group analysis of covariance (ANCOVA) with age and years of education as covariates. All reported $P$ values are Bonferroni corrected for multiple testing (four working memory tasks). Significant group differences were found in three of the four working memory tasks (articulatory rehearsal: $F=4.2 ; d f=4,32 ; P=0.031$; visuospatial rehearsal: $F=5.1 ; d f=4,32 ; P=0.01 ;$ visuospatial suppression: $F=4.5 ; d f=4 ; 32 ; P=0.022$ ).

In order to investigate these significant effects in more detail, we conducted further statistical analyses (ANCOVA adjusted for age and years of education) with regard to the separate factors 'familial loading' and 'diagnosis'.

\section{Verbal working memory (articulatory rehearsal)}

Within the schizophrenia group there was a significant influence of the factor 'familial loading'. Schizophrenic patients from multiply affected families performed worse in comparison to those from uniaffected families $(F=7.7$; $d f=1,12 ; \quad P=0.034)$. Group comparison between schizophrenic patients with familial loading and the matched sample of healthy controls revealed a significantly worse performance in the patients' group $(F=15.8$; $d f=1,12 ; P=0.004)$, whereas there were no significant differences between matched controls and schizophrenic patients without familial loading (see Fig. 1). Comparing the psychopathology scores within the schizophrenia group, there were no significant differences between the group with and without familial loading (see Table 3).

In contrast to these findings in the schizophrenia group, no significant influence of familial loading could be observed in schizoaffective patients, and there were no significant differences in task performance compared to healthy controls, neither in the group with nor in the group without familial loading.

The diagnostic specificity of the familial loading effect on performance in the articulatory rehearsal task was confirmed by a significant diagnosis $\times$ familial loading interaction for this working memory task (ANCOVA; $F=8.4 ; d f=1,32 ; P=0.026$ ).

\section{Visuospatial working memory}

There was a significant main effect for the factor 'familial loading' in the visuospatial rehearsal task $(F=8.8$; $d f=2,34 ; P=0.003$ ) and the visuospatial suppression task $(F=5.8 ; \quad d f=2,34 ; \quad P=0.027)$. Within the schizophrenia group, there were statistical trends for both visuospatial tasks with respect to the factor 'familial loading'. Patients from multiply affected families 
Fig. 1 Working memory task performance of schizophrenic patients with and without familial loading in comparison to healthy control subjects in four different working memory tasks. $* P<0.05$, $* * P<0.01$; ${ }^{+} P<0.1$

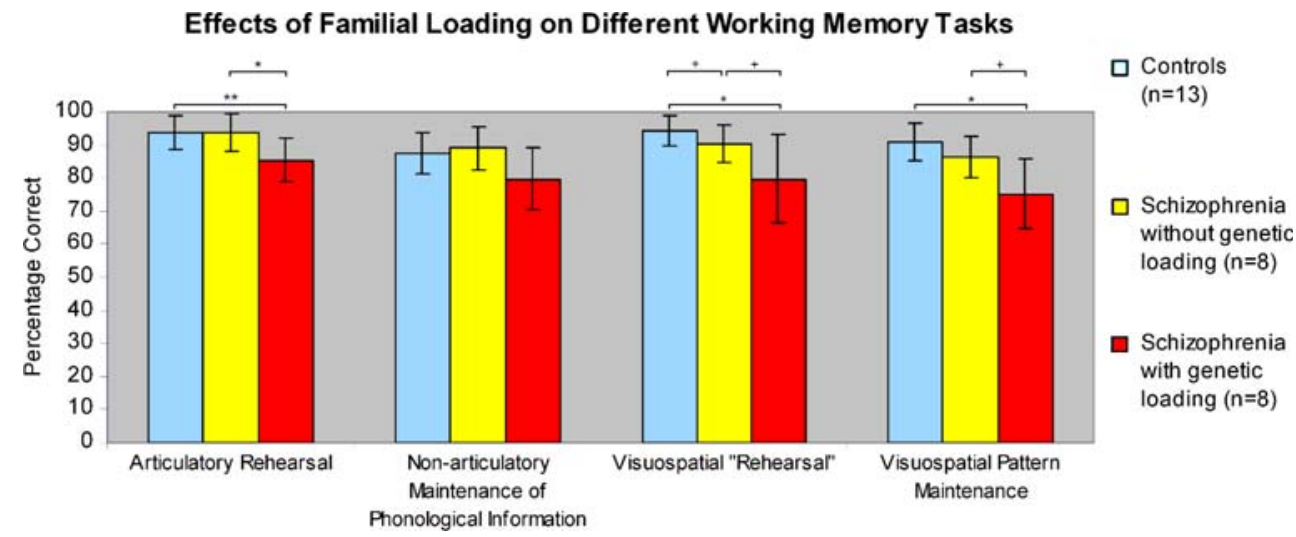

Table 3 Psychopathology scores

\begin{tabular}{llccc}
\hline & CGI & MADRS & PANSS-positive & PANSS-negative \\
\hline Schizophrenia with genetic loading & 4.25 & 14.38 & 11.5 & 13.0 \\
Schizophrenia without genetic loading & 4.63 & 13.5 & 12.5 & 14.63 \\
$P$ & 0.375 & 0.836 & 0.656 & 0.468 \\
\hline
\end{tabular}

performed worse than those from uniaffected families (visuospatial rehearsal: $F=5.6 ; d f=1,12 ; P=0.072$; visuospatial suppression: $F=5.0 ; d f=1,12 ; P=0.092$ ). Group comparison between schizophrenic patients with familial loading and the matched control group revealed a significantly worse performance in the patients' group (visuospatial rehearsal: $F=11.9 ; d f=1,12 ; P=0.01$; visuospatial suppression: $F=11.9 ; d f=1,12 ; P=0.01$ ). For schizophrenic patients without familial loading, there were no differences in comparison to healthy controls, but only a statistical trend for a worse performance in the visuospatial rehearsal task $(F=5.2 ; d f=1, \quad 12$; $P=0.082$ )

In the schizoaffective disorder group, no significant influence of the factor 'familial loading' could be observed. Compared to the control group, there were no significant differences in visuospatial task performance. There was only a statistical trend for the visuospatial pattern maintenance task, with schizoaffective patients without familial loading performing worse than healthy controls $(F=8.1$; $d f=1,6 ; P=0.059)$.

\section{Power calculations}

Power calculations were performed using (1) the mean values and standard deviations of performance rates as determined in the control sample, (2) group differences of $10 \%$ (which is conservative given the group differences of $11-13 \%$ observed in the preceding studies (e.g., Gruber et al. [18]), and (3) an alpha value of 0.05. For the analyses in schizophrenic patients ( 8 vs. 8 ), the power was 0.98 for the articulatory rehearsal task, 0.89 for the non-articulatory phonological maintenance task, 0.99 for visuospatial "rehearsal" and 0.94 for visuospatial pattern maintenance. For the smaller subgroups of schizoaffective patients (5 vs. $5)$ the respective power values were $0.91,0.73,0.95$ and 0.82 . This suggests that only for the schizoaffective patients and the non-articulatory phonological maintenance task there was not enough power to study the effects of familial loading, whereas the power was sufficient for all (diagnosis-specific) effects reported in this manuscript.

\section{Discussion}

In the present study, we investigated the influence of familial loading on working memory task performance in schizophrenic and schizoaffective patients. As working memory is considered to represent a promising endophenotype of schizophrenia, we expected that particularly schizophrenic patients from multiply affected families would show reduced performance in verbal and visuospatial working memory tasks as compared to patients from uniaffected families due to the higher degree of genetic loading. Consistent with this hypothesis, schizophrenic patients with additional familial loading performed worse in a verbal working memory task requiring the articulatory rehearsal mechanism compared to those without familial loading. No such effect was found for the schizoaffective patients. A significant interaction between familial loading and diagnosis confirmed this diagnosis-specific finding, suggesting a specific verbal working memory deficit in schizophrenic patients with familial loading. Since in a prior study working memory performance did not show any 
correlation with the patients' current psychopathology [25], the observed working memory deficits appear to be trait markers, which are relatively independent of the current state of the disease. Rather, a strong influence of the underlying neurobiology and genetics can be assumed. Our finding of a diagnosis-specific effect of familial loading on the performance of schizophrenic patients in the articulatory rehearsal task is in line with previous studies, which provided evidence for (1) the existence of specific subgroups of schizophrenic patients with selective working memory impairments (in particular with isolated deficits of the articulatory rehearsal mechanism while visuospatial working memory performance was spared [17]), and (2) for a role of articulatory rehearsal deficits in differentiating between groups of schizophrenic and schizoaffective patients (the latter performing well in articulatory rehearsal tasks) [18]. Together, these findings suggest a strong influence of familial loading with (and the genetic risk for) schizophrenia on the functional integrity of the articulatory rehearsal mechanism of verbal working memory. This further supports the assumption that dysfunctions of the neural system underlying articulatory rehearsal may qualify as a promising endophenotype of schizophrenia [17, $18]$.

With respect to visuospatial working memory, we observed a statistical trend in the same direction; schizophrenic patients with familial loading performed worse than those without. For the visuospatial rehearsal task, we found a significant main effect for the factor 'familial loading', however, without a significant interaction with diagnosis. These results are still compatible with the hypothesis, that visuospatial working memory may also be influenced by genetic factors and may also represent an endophenotype for schizophrenia. However, the fact that there was also a statistical trend for reduced performance of schizophrenic patients without familial loading as compared to healthy controls suggests that visuospatial working memory deficits in schizophrenia may also occur independent from familial/genetic loading.

Within the group of schizoaffective patients, we did not find a significant influence of familial loading on working memory performance, neither in the verbal nor in the visuospatial tasks. This negative finding appears to partly contrast with a series of studies reporting cognitive deficits in schizoaffective patients resembling those in patients with schizophrenia $[6,7,12,20,21]$. However, the literature concerning this issue is inconsistent as other researchers in fact reported evidence for differential cognitive deficits between schizophrenia and schizoaffective disorder. For instance, Stip [23] observed significant differences on motor screening and explicit memory tests where schizoaffective patients performed better, while Gruber [18] identified the articulatory rehearsal mechanism in verbal working memory as a potential endophenotype differentiating between the two diagnoses. The apparent inconsistencies between these findings of prior studies could in part be explained by the lack of specificity in neurofunctional testing in some of the studies and by the pathogenetic and pathophysiological heterogeneity that may exist within psychiatric diagnoses according to current classification systems. The results of the present study provide further evidence for a differential pattern of working memory dysfunction between schizophrenic and schizoaffective patients, which could be related to differences in the underlying pathophysiology.

Although our power calculations revealed that the statistical power was sufficient for almost all subgroup comparisons in the different working memory task (except for the schizoaffective subgroups in the nonarticulatory phonological maintenance task; see Sect. "Results"), the present findings are certainly limited by the relatively small sample sizes and, therefore, should be confirmed in future studies with larger samples. Nevertheless, the fact that in spite of the small sample sizes there were significant effects of the factor 'familial loading' with respect to working memory functioning in schizophrenic patients suggests a high impact of familial (and genetic) loading for schizophrenia on working memory functioning. Thus, our results further confirm previous findings of a decline in working memory test performance depending on the degree of shared genes $[3$, $10,24]$.

In the present study, this genetic influence was observed specifically in the group of schizophrenic patients, and predominantly in the articulatory rehearsal task. These findings are promising and should encourage future studies to further investigate genetic influences on the different neural sub-systems of human working memory in schizophrenia. On this way, the different patterns of working memory deficits described above could prove helpful to characterize and differentiate subgroups of schizophrenic patients with a more homogeneous underlying pathophysiology. Moreover, although a genetic impact on working memory functioning is evident, the specific genes contributing to this effect are not yet identified. Further studies should address this issue and seek to identify gene loci or polymorphisms associated with interindividual variation of working memory performance in the general population. According to the assumption that working memory impairment as an intermediate phenotype of schizophrenia plays a role in the underlying pathophysiology of the disorder, these genes could possibly also represent susceptibility genes for schizophrenic psychoses. 
Open Access This article is distributed under the terms of the Creative Commons Attribution Noncommercial License which permits any noncommercial use, distribution, and reproduction in any medium, provided the original author(s) and source are credited.

\section{References}

1. Albus M, Hubmann W, Mohr F, Hecht S, Hinterberger-Weber P, Seitz NN, Küchenhoff H (2006) Neurocognitive functioning in patients with first-episode schizophrenia: results of a prospective 5-year follow-up study. Eur Arch Psychiatry Clin Neurosci 256:442-451

2. Barrantes-Vidal N, Aguilera M, Campanera S, Fatjó-Vilas M, Guitart M, Miret S, Valero S, Fañanás L (2007) Working memory in siblings of schizophrenia patients. Schizophr Res 95:76-85

3. Cannon TD, Huttunen MO, Lonnqvist J et al (2000) The inheritance of neuropsychological dysfunction in twins discordant for schizophrenia. Am J Hum Genet 67:369-382

4. Carter C, Robertson L, Nordahl T, Chaderjian M, Kraft L, O'Shora-Celaya L (1996) Spatial working memory deficits and their relationship to negative symptoms in unmedicated schizophrenia patients. Biol Psychiatry 40:930-932

5. Conklin HM, Curtis CE, Calkins ME, Iacono WG (2005) Working memory functioning in schizophrenia patients and their first-degree relatives: cognitive functioning shedding light on etiology. Neuropsychologia 43:930-942

6. Evans JD, Heaton RK, Paulsen JS, McAdams LA, Heaton SC, Jeste DV (1999) Schizoaffective disorder: a form of schizophrenia or affective disorder? J Clin Psychiatry 60:874-882

7. Fiszdon JM, Richardson R, Greig T, Bell MD (2007) A comparison of basic and social cognition between schizophrenia and schizoaffective disorder. Schizophr Res 91:117-121

8. Fleming K, Goldberg TE, Gold JM, Weinberger DR (1995) Verbal working memory dysfunction in schizophrenia: use of a Brown-Peterson paradigm. Psychiatry Res 56:155-161

9. Forbes NF, Carrick LA, McIntosh AM, Lawrie SM (2008) Working memory in schizophrenia: a meta-analysis. Psychol Med 2008 Oct 23:1-17 [Epub ahead of print]

10. Glahn DC, Therman S, Manninen M et al (2003) Spatial working memory as an endophenotype for schizophrenia. Biol Psychiatry 53:624-626

11. Gonzalez-Blanch C, Alvarez-Jimenez M, Rodriguez-Sanchez JM, Perez-Iglesias R, Vazquez-Barquero JL, Crespo-Facorro B (2006) Cognitive functioning in the early course of first-episode schizophrenia spectrum disorders: timing and patterns. Eur Arch Psychiatry Clin Neurosci 256:364-371

12. Gooding DC, Tallent KA (2002) Spatial working memory performance in patients with schizoaffective psychosis versus schizophrenia: a tale of two disorders? Schizophr Res 53:209_ 218
13. Gottesman II, Gould TD (2003) The endophenotype concept in psychiatry: etymology and strategic intentions. Am J Psychiatry 160:636-645

14. Gruber O (2001) Effects of domain-specific interference on brain activation associated with verbal working memory task performance. Cereb Cortex 11:1047-1055

15. Gruber O, von Cramon DY (2003) The functional neuroanatomy of human working memory revisited. Evidence from 3-T fMRI studies using classical domain-specific interference tasks. Neuroimage 19:797-809

16. Gruber O, Goschke T (2004) Executive control emerging from dynamic interactions between brain systems mediating language, working memory and attentional processes. Acta Psychol 115:105-121

17. Gruber O, Gruber E, Falkai P (2005) Neural correlates of working memory deficits in schizophrenic patients. Ways to establish neurocognitive endophenotypes of psychiatric disorders. Radiologe 45:153-160

18. Gruber O, Gruber E, Falkai P (2006) Articulatory rehearsal in verbal working memory: a possible neurocognitive endophenotype that differentiates between schizophrenia and schizoaffective disorder. Neurosci Lett 405:24-28

19. Maier W (2006) Do schizoaffective disorders exist at all? Acta Psychiatr Scand 113:369-371

20. Manschreck TC, Maher BA, Beaudette SM, Redmond DA (1997) Context memory in schizoaffective and schizophrenic disorders. Schizophr Res 26:153-161

21. Miller LS, SwansonGreen T, Moses JA, Faustman WO (1996) Comparison of cognitive performance in RDC-diagnosed schizoaffective and schizophrenic patients with the Luria-Nebraska neuropsychological battery. J Psychiatr Res 30:277-282

22. Spellmann I, Müller N, Musil R, Zill P, Douhet A, Dehning S, Cerovecki A, Bondy B, Möller HJ, Riedel M (2008) Associations of SNAP-25 polymorphisms with cognitive dysfunctions in Caucasian patients with schizophrenia during a brief trail of treatment with atypical antipsychotics. Eur Arch Psychiatry Clin Neurosci 258:335-344

23. Stip E, Sepehry AA, Prouteau A, Briand C, Nicole L, Lalonde P, Lesage A (2005) Cognitive discernible factors between schizophrenia and schizoaffective disorder. Brain Cogn 59:292-295

24. Tuulio-Henriksson A, Arajarvi R, Partonen T et al (2003) Familial loading associates with impairment in visual span among healthy siblings of schizophrenia patients. Biol Psychiatry 54:623-628

25. Zilles D, Gruber E, Falkai P, Gruber O (2005) Funktionelle Integrität neuronaler Netzwerke mit Arbeitsgedächtnisfunktion bei schizophrenen Psychosen. Nervenarzt 76(S1):S33-S34 